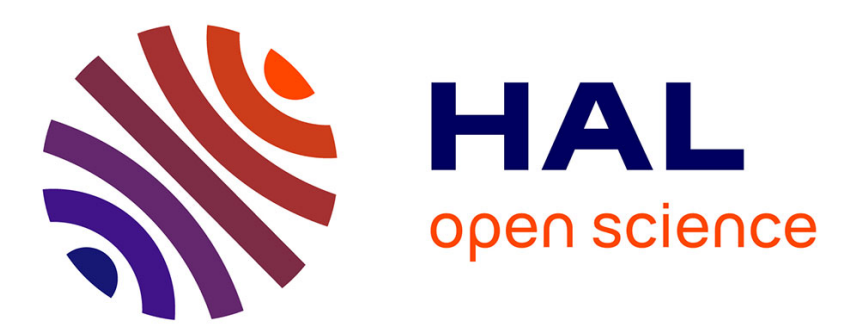

\title{
Direction finding of half-gyrofrequency VLF emissions in the off-equatorial region of the magnetosphere and their generation and propagation
}

\author{
H. Muto, M Hayakawa, Michel Parrot, François Lefeuvre
}

\section{- To cite this version:}

H. Muto, M Hayakawa, Michel Parrot, François Lefeuvre. Direction finding of half-gyrofrequency VLF emissions in the off-equatorial region of the magnetosphere and their generation and propagation. Journal of Geophysical Research, 1987, 92 (A7), pp.7538. 10.1029/JA092iA07p07538 . insu-03125005

\section{HAL Id: insu-03125005 \\ https://hal-insu.archives-ouvertes.fr/insu-03125005}

Submitted on 29 Jan 2021

HAL is a multi-disciplinary open access archive for the deposit and dissemination of scientific research documents, whether they are published or not. The documents may come from teaching and research institutions in France or abroad, or from public or private research centers.
L'archive ouverte pluridisciplinaire HAL, est destinée au dépôt et à la diffusion de documents scientifiques de niveau recherche, publiés ou non, émanant des établissements d'enseignement et de recherche français ou étrangers, des laboratoires publics ou privés. 


\title{
Direction Finding of Half-Gyrofrequency VLF Emissions in the Off-Equatorial Region of the Magnetosphere and Their Generation and Propagation
}

\author{
H. Muto and M. Hayakawa ${ }^{1}$ \\ Research Institute of Atmospherics, Nagoya University, Toyokawa, Aichi, Japan \\ M. Parrot AND F. LefeuVre \\ Laboratoire de Physique et Chimie de l'Environnement, Centre National de la Recherche Scientifique \\ Orleans, France
}

\begin{abstract}
The spectra of VLF/ELF emissions in the off-equatorial region in the outer magnetosphere are, on occasion, found to consist of upper and lower band's with a frequency gap between them. The wave normal directions of the upper band VLF emissions have been determined by means of wave distribution function analyses based first on the measurement of three magnetic field components only, and then with the additional use of an electric field component. It is found that the wave distribution functions are composed of two peaks whose central wave normal angles $\theta$ with respect to the magnetic field at a geomagnetic latitude of $\sim 17^{\circ}$ are close to the local oblique resonance cone $\theta_{\text {res }}$ but whose values of $\theta$ are always about $15^{\circ}-20^{\circ}$ less than $\theta_{\text {res }}$ at a higher latitude of $\sim 26^{\circ}$. This observed wave normal behavior is compared with the theoretical prediction from direct ray tracing studies using the hypothesis deduced from a previous investigation that the waves are generated at the equator with wave normals close to $\theta_{\text {res: }}$ A combination of this comparison and inverse ray tracing in which the initial wave normal directions are those determined from the wave distribution analysis has yielded the result that the $\dot{\theta}$ values of the upper band VLF emissions are very close to $\theta_{\text {res }}$ at the equator and that the normalized frequency there, $\Lambda_{\mathrm{eq}}$, is above 0.5 . Hence the upper band VLF emissions are identified as being "half-gyrofrequency" VLF emissions generated in the vicinity of the magnetic equator, with their wave normals close to $\theta_{\text {res. }}$. The characteristics of propagation between the equatorial source region and the observing positions have been investigated by direct ray tracing. The generation mechanism is also discussed.
\end{abstract}

\section{INTRODUCTION}

In addition to strong electrostatic "odd half-harmonic emissions" [Kennel et al., 1970; Kennel and Ashour-Abdalla, 1982], VLF emissions with frequencies above half the electron gyrofrequency $\left(\omega / \omega_{H} \geq 0.5\right.$ where $\omega$ is wave frequency and $\omega_{H}$ is electron gyrofrequency), called "half-gyrofrequency emissions," have occasionally been observed by satellites near the geomagnetic equator in the magnetosphere beyond the plasmapause by Coroniti et al. [1971], Tsurutani and Smith [1974], Burtis and Helliwell [1976], Maeda [1976], and Hayakawa et al. [1984]. All of these except the last were unable to specify the wave model (electromagnetic or electrostatic), but several authors have speculated about generation mechanisms. Recently, Hayakawa et al. [1984] have elucidated the detailed characteristics of the emissions by determining the wave normal directions of the waves observed in the vicinity of the equator on the GEOS 2 satellite. They found that the wave normal directions of the emissions are very close to the oblique resonance cone $\theta_{\text {res }}$ for whistler mode propagation, which means that they are quasi-electrostatic whistler mode waves. This conclusion is supported by the measured ratio of the electric and the magnetic field intensity. On the basis of these results, Ohmi and Hayakawa [1986] have suggested an electrostatic instability in a realistic two-component mag-

\footnotetext{
${ }^{1}$ Also at Laboratoire de Physique et Chimie de l'Environnement, Centre National de la Recherche Scientifique, Orleans, France.

Copyright 1987 by the American Geophysical Union.

Paper number 6A8887.

0148-0227/87/006A-8887\$05.00
}

netospheric plasma consisting of cold electrons and populated low-energy (suprathermal) electrons of energy of the order of a few tens of electron volts and with a temperature anisotropy sufficient to drive an instability. From the work by Hayakawa et al. [1984] the equatorial generation of these emissions is to be expected with the lowest frequency produced being nearly coincident with one half of the electron gyrofrequency near the equator. In order to make further studies of the generation and propagation of these waves, it is highly desirable to investigate observations in the off-equatorial regions of the magnetosphere.

The present paper is complementary to the previous equatorial study of Hayakawa et al. [1984] and presents the characteristics and the direction-finding results of supposedly half-gyrofrequency VLF emissions observed by the GEOS 1 satellite at two different geomagnetic latitudes in the offequatorial region of the magnetosphere. It follows the approach adopted by Lefeuvre and Helliwell [1985] for hiss emissions. The observed properties of wave normal directions in the off-equatorial regions are first compared to results obtained by three-dimensional ray tracing performed with values of the plasma and wave parameters similar to those encountered on GEOS 2 [Hayakawa et al., 1984]. The waves are supposed to be half-gyrofrequency VLF emissions generated at the equator, with their wave normals close to $\theta_{\text {res }}$. Then inverse ray tracings are conducted from the observation points toward the equator, starting with the wave normals determined in the off-equatorial regions. The wave normals at the equator are compared with the previous equatorial observations by Hayakawa et al. [1984] and with the theoretical study by Ohmi and Hayakawa [1986]. These ray tracing studies in 
TABLE 1. Analyzed Events

\begin{tabular}{|c|c|c|c|c|c|c|c|}
\hline \multirow[b]{2}{*}{ Date } & \multirow[b]{2}{*}{ UT } & \multicolumn{2}{|c|}{ Satellite Location } & \multicolumn{2}{|c|}{ Plasma Parameter } & \multirow[b]{2}{*}{ MLT } & \multirow[b]{2}{*}{$K p$} \\
\hline & & $R, R_{E} ; \Lambda_{m}, \operatorname{deg}$ & $L$ Value & $f_{H}, \mathrm{kHz}$ & $f_{p}, \mathrm{kHz}$ & & \\
\hline Oct. 12,1977 & 0652 & $6.93 ; 17.4$ & 7.60 & 2.711 & 10.9 & 1005 & 5 \\
\hline Sept. 19, 1977 & 1148 & $6.84 ; 26.2$ & 8.00 & 3.490 & 19.2 & 1202 & 4 \\
\hline
\end{tabular}

combination with the direction-finding measurements enable us to investigate (1) whether half-gyrofrequency emissions are generated only in the vicinity of the geomagnetic equator, (2) whether their wave normals at the source region are close to $\theta_{\text {res }}$, and (3) the subsequent propagation if the generation region is considered to be at the equator.

\section{Data Source and Direction-Finding METHOD}

The field data are signals obtained by the S-300 experiment on board the GEOS 1 satellite which measures continuously the electric and magnetic components of the wave field. The received signals are subjected to on-board analyses by the sweep frequency analyzers (SFAs) and the correlator. Six SFAs, which may be connected to any sensor combination, have a bandwidth of $300 \mathrm{~Hz}$ and are swept in frequency in the range 0-77 kHz. Before being telemetered to the ground, the signals are transposed in frequency, passed through identical low-pass filters at $450 \mathrm{~Hz}$, and sampled at $1.488 \mathrm{kHz}$. The correlator output, after suitable Fourier transformation on the ground, yields a spectrum of bandwidth $2.5 \mathrm{kHz}$ during the ELF part of a special survey mode or $5.0 \mathrm{kHz}$ during the VLF part. The orbit of the satellite is given by Knott [1978], and a detailed description of the GEOS S-300 experiments is given by Jones [1978] and $S-300$ Experimenters [1979].

The spectral matrix at each Fourier component is estimated by using the SFA magnetic data during the VLF part. Several direction-finding methods have been proposed (see Lefeuvre et al. [1981, 1982], for example), but in the present paper we utilize only the wave distribution function (WDF) method in which the maximum entropy concept is applied to the measured spectral matrices [Lefeuvre et al., 1981, 1982; Hayakawa et al., 1986]. In order to illustrate the direction-finding results, we adopt a Cartesian coordinate system Oxyz where the $z$ axis is parallel to the earth's magnetic field $B_{0}$, the $x$ axis is in the magnetic meridian plane and is directed toward the earth, and the $y$ axis completes the orthogonal set and is directed westward. The wave normal direction $\mathbf{k}$ is characterized by the polar angle $\theta$ between $\mathbf{k}$ and $\mathbf{B}_{0}$ and by the azimuthal angle $\phi$ with respect to $O x$. It should be noted that this Cartesian coordinate is different from the one in the work by Lefeuvre et al. [1982] and Hayakawa et al. [1984].

\section{Wave Spectra and Wave Normal. Directions OF THE EMISSIONS OBSERVED IN THE OfF-Equatorial Region OF THE MAGNETOSPHERE}

After looking at many VLF spectrograms from the GEOS 1 satellite, we have selected two events with intensity sufficient for direction finding and at different geomagnetic latitudes. These events are listed in Table 1 . The event of October 12, 1977 , is observed at the smaller geomagnetic latitude $\Lambda_{m}$ of $17.4^{\circ}$, whereas the event of September 19,1977 , is recorded at the higher latitude, $\Lambda_{m}=26.2^{\circ}$. In the table are listed the $L$ value and plasma parameters (electron gyrofrequency $f_{H}\left(=\omega_{H} / 2 \pi\right)$ and electron plasma frequency $\left.f_{p}\right)$. The two events were observed before or around local noon. The geomagnetic activity $K p$ is also indicated. The wave characteristics for these events are described below.

\subsection{The Event of October 12, 1977}

Figure 1 illustrates the ELF spectrogram of VLF/ELF emissions in a range up to $2.5 \mathrm{kHz}$ encompassing the time during which the direction-finding measurement was made, and it indicates that VLF emissions are composed of two bands separated by a frequency gap at $\sim 1.0 \mathrm{kHz}$. The frequency gap is much smaller than the half-gyrofrequency $\left(f_{H} /\right.$ $2=1.356 \mathrm{kHz}$ ) at the observing point. The lower band is very intense and mainly consists of structured chorus emissions. The upper band VLF emissions are relatively weak; this band consists of only a few structured elements. If we suppose that the generation mechanism as proposed by Ohmi and Hayakawa [1986] is operative for the upper band emissions at the satellite position, the wave frequency to be excited must be higher than the local $f_{H} / 2$. This prediction is in obvious disagreement with the observation, which is suggestive of the source being located at a region with smaller geomagnetic field. These upper band emissions will later be shown to be half-gyrofrequency VLF emissions at the equator.

The direction-finding computation was attempted for several time intervals and frequencies, but mainly because of the weak signal strength, only a few cases were successful. An example of one such WDF is illustrated in Figure 2, which corresponds to the upper band VLF emission at a specific frequency of $1057 \mathrm{~Hz}$. The power spectral density for this, the strongest event, is only about $2.0 \times 10^{-5} \gamma^{2} / \mathrm{Hz}$. The WDF is presented in the form of contours of wave energy density in a polar diagram with a linear scale of wave energy running from 0 to 10 . In the figure we have only illustrated the part of the solution in the range of $0 \leqq \theta \leqq \pi / 2$. The outermost circle corresponds to $\theta=90^{\circ}$, while the circle drawn as a heavy line indicates the position of the oblique resonance cone $\theta_{\text {res }}$ for whistler model propagation ( $\cos \theta_{\text {res }}=\omega / \omega_{H}$ [Helliwell, 1965]). We have confirmed, using the detailed gray-scale spectrograms, that the relevant frequency is above the frequency gap at the time in question. The gray scale displays are used for all the data used in the presont paper for examining the spectral shapes and the location of the frequency gap. In the figure caption we have indicated the stability parameter $Q$ and prediction parameter $P_{r}$, which are the indices of numerical stability of the solution. When $Q \lesssim 1$, the solution is considered to be stable, and for $P_{r} \simeq 1$, the data are considered to be correctly fitted (see Lefeuvre et al. $[1981,1982]$ for further details). The information on the three eigenvalues $\left(\lambda_{1}>\lambda_{2}>\right.$ $\lambda_{3}$ ) of the measured spectral matrix is also given [Buchalet and Lefeuvre, 1981; Hayakawa et a!., 1984, 1986].

The WDF in Figure 2 is seen to exhibit two peaks, completely separated from each other, their $\phi$ being separated by about $180^{\circ}$. The wave energy associated with the main peak is 


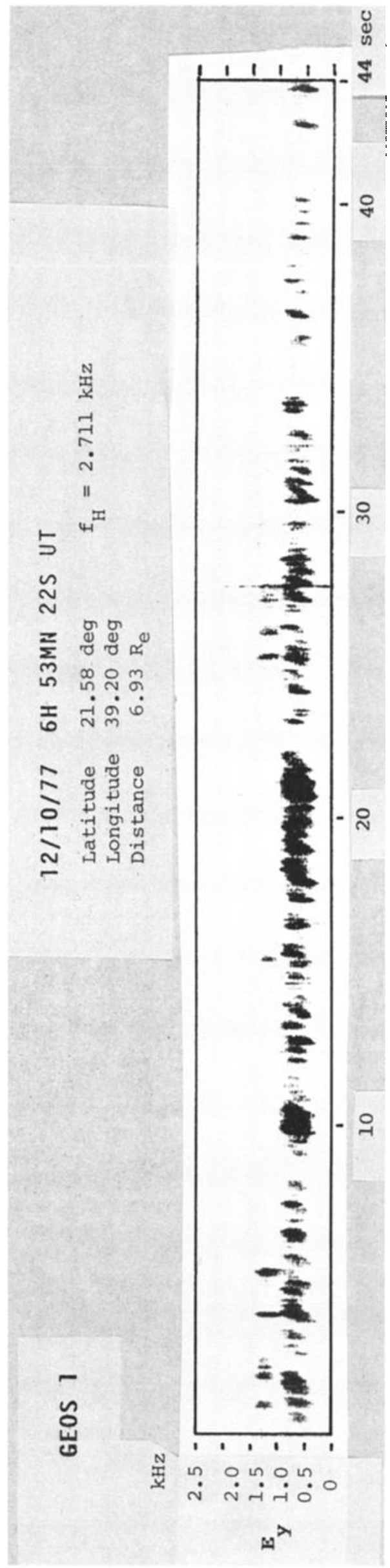

in close proximity to the region of $\theta=\theta_{\text {res }}$ over a wide range, of the order of $\sim 45^{\circ}$, in $\phi$. The $\theta$ value of the main peak is about $14^{\circ}$ from the circle of $\theta_{\text {res }}$. The $\theta$ value of the secondary peak is much closer to $\theta_{\text {res }}$. Another reliable WDF has been obtained for this event with intensity $\sim 10^{-5} \gamma^{2} / \mathrm{Hz}$, which also shows that the WDF is doubly peaked, but with both peaks being much closer to $\theta_{\text {res }}$.

\subsection{The Event of September 19, 1977}

Two successive examples of the spectrograms of VLF/ELF emissions during $88 \mathrm{~s}$ starting at 1148:14 UT are presented in Figures $3 a$ and $3 b$. These are quite different from the previous event (see Figure 1). The spectrograms are clearly composed of upper and lower bands which are equally intense (more than $10^{-3} \gamma^{2} / \mathbf{H z}$ ) and which overlap on some occasions. At a later time in Figure $3 b$ the upper band becomes the more intense. The frequency gap inferred from the spectrograms is around $1.3 \mathrm{kHz}$, which is again much less than the half-gyrofrequency $(1.745 \mathrm{kHz})$ at the observing point. The emissions in the lower band are found to be clearly structured: in this case, rising tones or chorus. The frequency spectrum of the upper band is quasi-continuous, more similar to hiss. Its bandwidth is found to be of the same magnitude as observed at the equator [Hayakawa et al., 1984], which is much larger than the bandwidth given by Coroniti et al. [1971].

The determination of WDFs has been performed over a time interval of $1 \mathrm{~min}$ starting at 1148:58 UT, and at several other time intervals and frequencies. The total number of the estimated WDFs was 15. Four examples are presented in Figures $4 a-4 d$, which are in the same format as Figure 2. Only the magnetic field components have been utilized in the analyses, which results in there being an ambiguity in the sense of propagation [Lefeuvre et al., 1981; Hayakawa et al., 1984, 1986]. Again, only the sector $0 \leqq \theta \leqq \pi / 2$ is illustrated in each figure. The WDFs in Figure 4 are seen to be double-peaked. Among them the two peaks in Figures $4 a, 4 b$, and $4 d$ are completely detached, while the two peaks in Figure $4 c$ are not so well separated from each other. The characteristics of the main peaks in the figures are quite similar, particularly in Figures $4 a, 4 b$, and $4 c$. Their central $\theta$ values are about $15^{\circ}-20^{\circ}$ inside the $\theta_{\text {res}}$, and their $\phi$ values are not so far from each other as in Figure 2 . It is difficult to know whether the $\sim 30^{\circ}$ rotation in $\phi$ of the main peak in Figure $4 d$ as compared to Figures $4 a-4 c$ is due to errors in the data caused mainly by spectral analysis or to a different source-satellite geometry. The intensity and $\phi$ value of the secondary peaks are quite variable. There is a strong similarity between Figures $4 b$ and $4 c$, on the one hand, and between Figures $4 a$ and $4 d$, on the other hand. In conclusion, we can say that the propagation characteristics of the hiss-type emissions of the upper band during the 1-min interval show features which seem constant in time. The wave energy is mainly conveyed by the waves whose wave normal directions have $\theta$ values relatively close to the resonance cone $\left(15^{\circ}-20^{\circ}\right.$ inside $\left.\theta_{\text {res }}\right)$ and $\phi$ values around $90^{\circ}$ (the $k$ vectors are $\sim 90^{\circ}$ off the magnetic meridian plane). Some energy is also conveyed by waves whose wave normals have $\theta$ values as large as those of the main peaks but whose $\phi$ values are apparently random. The above $\theta$ characteristics are confined to be generally true for the whole of this event as can be seen in Figure 5a, which is based on the analysis of all the WDF computed. The occurrence histogram of $\phi$ including the main and secondary peaks is plotted in Figure $5 b$. It indicates that $\phi$ is distributed over the whole range $[-\pi, \pi]$, although there 


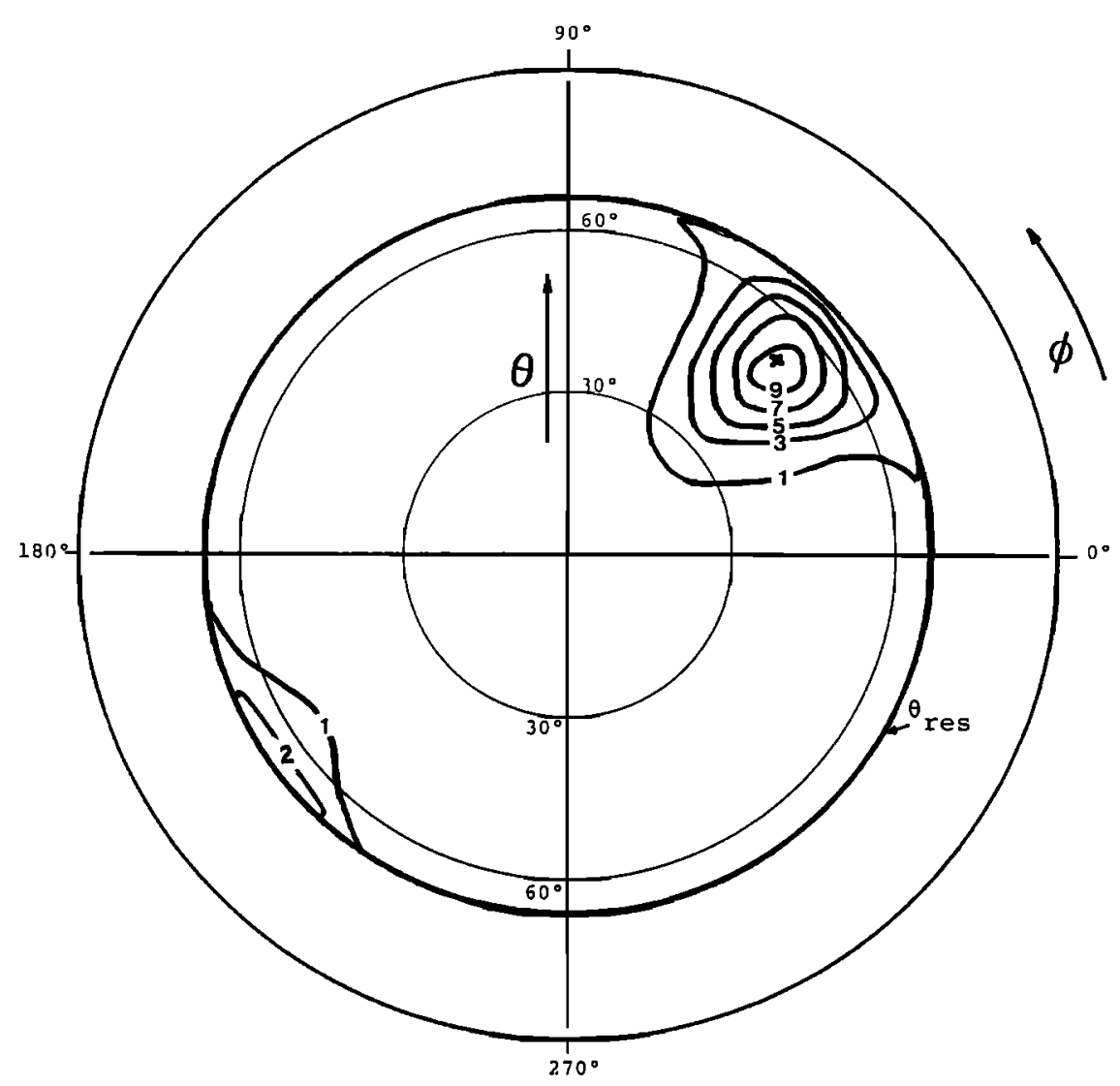

Fig. 2. The wave distribution functions (WDFs) in the form of contours of wave energy at $1057 \mathrm{~Hz}$ at $0655: 49.356 \mathrm{UT}$ on October 12, 1977. The scale of WDFs is linear and runs from 0 to 10 . The three eigenvalues $\left(\lambda_{1}>\lambda_{2}>\lambda_{3}\right)$ are estimated from the spectral matrix derived only from the magnetic field components $\left(\lambda_{2} / \lambda_{1}=0.12\right.$ and $\left.\lambda_{3} / \lambda_{2}=0.3\right)$. Also, the numerical stability of the solution is indicated by the two parameters, the stability parameter $Q$ and prediction parameter $P_{r}\left(Q=1.16\right.$ and $\left.P_{r}=2.65\right)$.

seem to be two occurrence peaks at $\phi \sim-135^{\circ}$ and $\phi \sim 75^{\circ}$, which may or may not be significant.

To remove the ambiguity in the sign of the $\mathbf{k}$ direction, further analyses have been attempted including one electric field component, $E_{y}$. Taking into account the uncertainty involved in estimating the coupling impedance between the electric antennas and the plasma (P. M. E. Decreau, private communication, 1979), the same criteria of acceptance of a solution have been used as were used by Parrot and Lefeuvre [1986]: (1) $Q \lessgtr 1$ and $P_{r} \sim 1$, (2) the solution obtained from three magnetic plus one electric data must be consistent with the solution derived from the magnetic field components alone, and (3) the number of sign differences between the polarity of the real and imaginary parts of the spectral matrix predicted from the solution and that of the measured spectral matrix should not be more than 2. (See Parrot and Lefeuvre ([1986] for further details.) As a result of analyses for all cases, no solution satisfying the above three criteria was found, mainly because the solutions were unstable. Despite this, the solutions seemed to indicate two peaks, main and secondary, propagating from lower to higher latitudes. This was based on the comparison of the ratio of the powers in the two hemispheres, $0 \leqq \theta \leqq \pi / 2$ and $\pi / 2 \leqq \theta \leqq \pi$ in each case. The fact that we are unsuccessful in having a distinct separation in the wave power propagating from and toward the equator seems to be due to the uncertainties in the electric field measurements.

\subsection{Summary of the Wave Properties Observed Away From the Equator}

The experimental study based on the observations at two geomagnetic latitudes $\left(\Lambda_{m}=17.4^{\circ}\right.$ and $\left.26.2^{\circ}\right)$ has yielded the following results.

1. The wave spectra at both geomagnetic latitudes are composed of two bands; the lower band consists of chorus emissions, and the upper band is the subject of the present paper. The spectra of the upper band emissions are not always smooth steady hiss hands. The emissions in Figure 3 are of the hiss type, but those in Figure 1 are structured and impulsive ones. These impulsive structures seem to be very different from the corresponding structures observed by Tsurutani and Smith [1974], who observed chorus with much smaller $d f / d t$ and falling tones. This variety of frequency spectra of the upper band emissions is similar in nature to odd half-harmonic electrostatic emissions [Kennel and Ashour-Abdalla, 1982].

2. The bandwidth of the upper band is $300-500 \mathrm{~Hz}$, which is nearly of the same magnitude as that observed previously at the equator [Hayakawa et al., 1984] but is much larger than that observed off the equator $\left(\Lambda_{m} \sim 10^{\circ}\right)$ by Coroniti et al. [1971].

3. The frequency gap between the two bands is found to be always much smaller than the local $f_{H} / 2$, which is indicative of the source being located at a position with smaller geomagnetic field. 

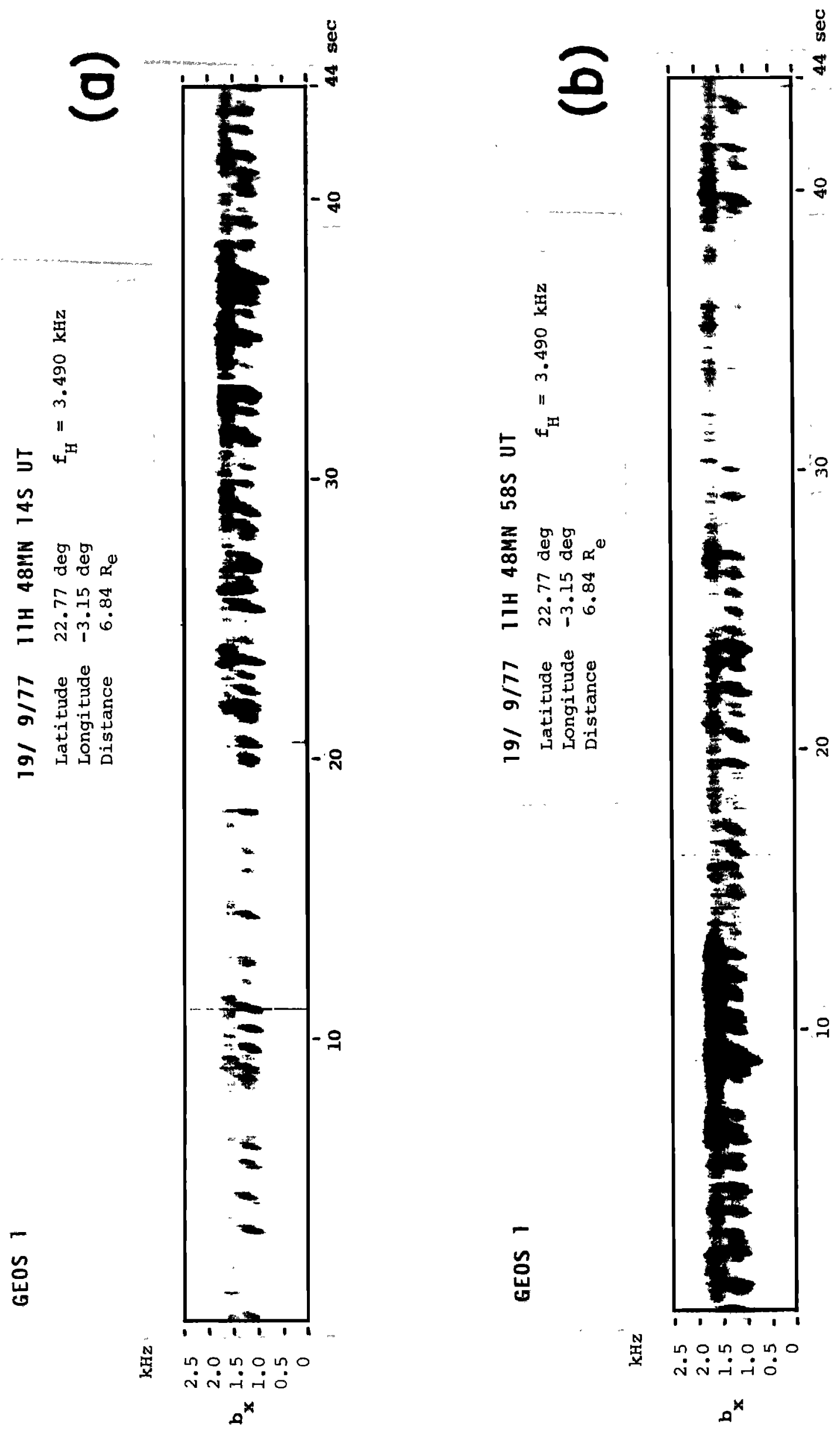

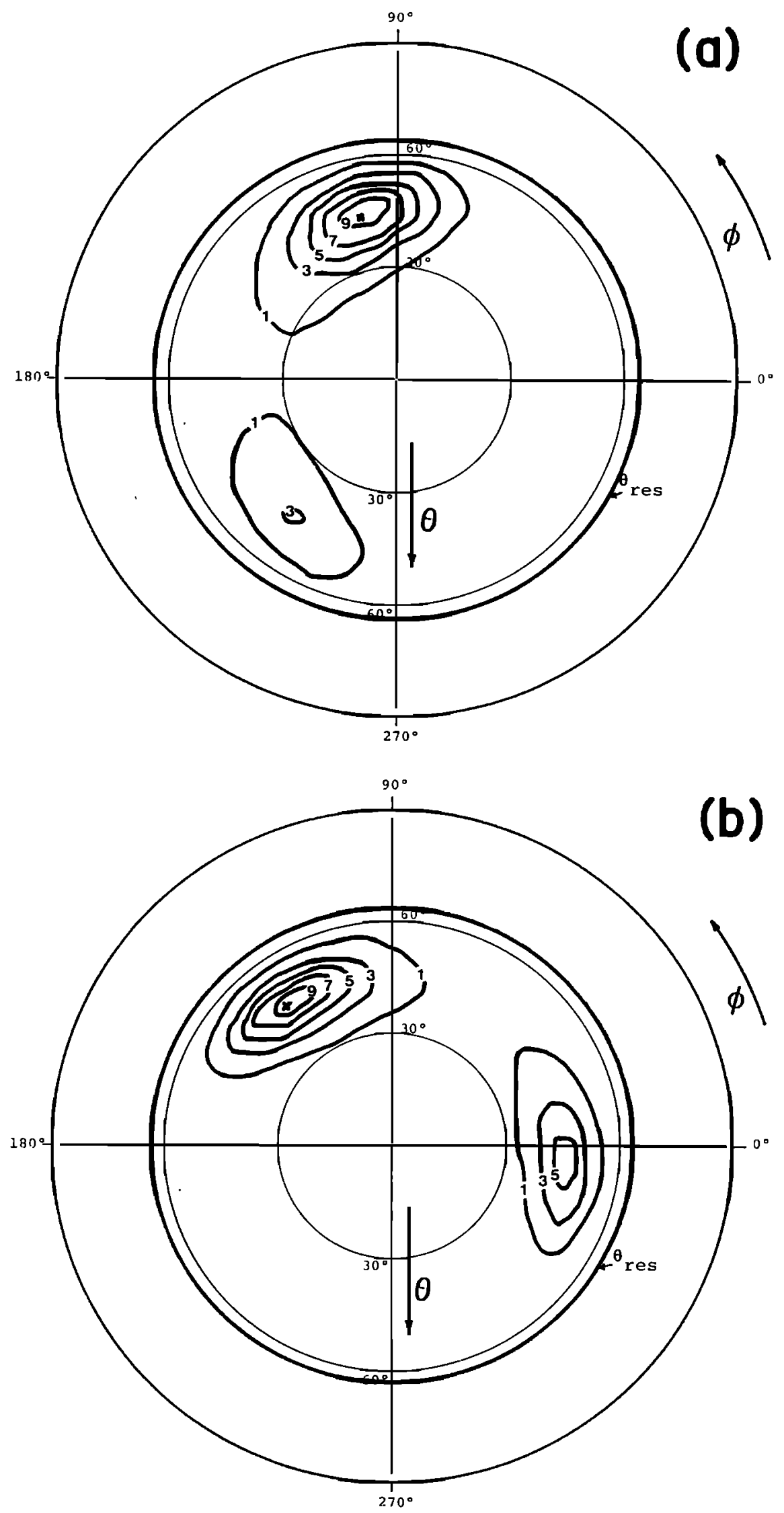

Fig. 4. WDFs on September 19, 1977: (a) $1148: 58.711 \mathrm{UT}, f=1510 \mathrm{~Hz}, \lambda_{2} / \lambda_{1}=0.203, \lambda_{3} / \lambda_{2}=0.032, Q=1.70$, and $P_{r}=0.97 ;(b) 1149: 07.827 \mathrm{UT}, f=1557 \mathrm{~Hz}, \lambda_{2} / \lambda_{1}=0.068, \lambda_{3} / \lambda_{2}=0.041, Q=1.07$, and $P_{r}=1.29 ;(c) 1149: 24.855 \mathrm{UT}$, $f=2009 \mathrm{~Hz}, \lambda_{2} / \lambda_{1}=0.112, \lambda_{3} / \lambda_{2}=0.142, Q=0.66$, and $P_{r}=0.88$; and $(d) 1149: 46.355 \mathrm{UT}, f=1916 \mathrm{~Hz}, \lambda_{2} / \lambda_{1}=0.215$, $\lambda_{3} / \lambda_{2}=0.063, Q=0.45$, and $P_{r}=0.69$. 

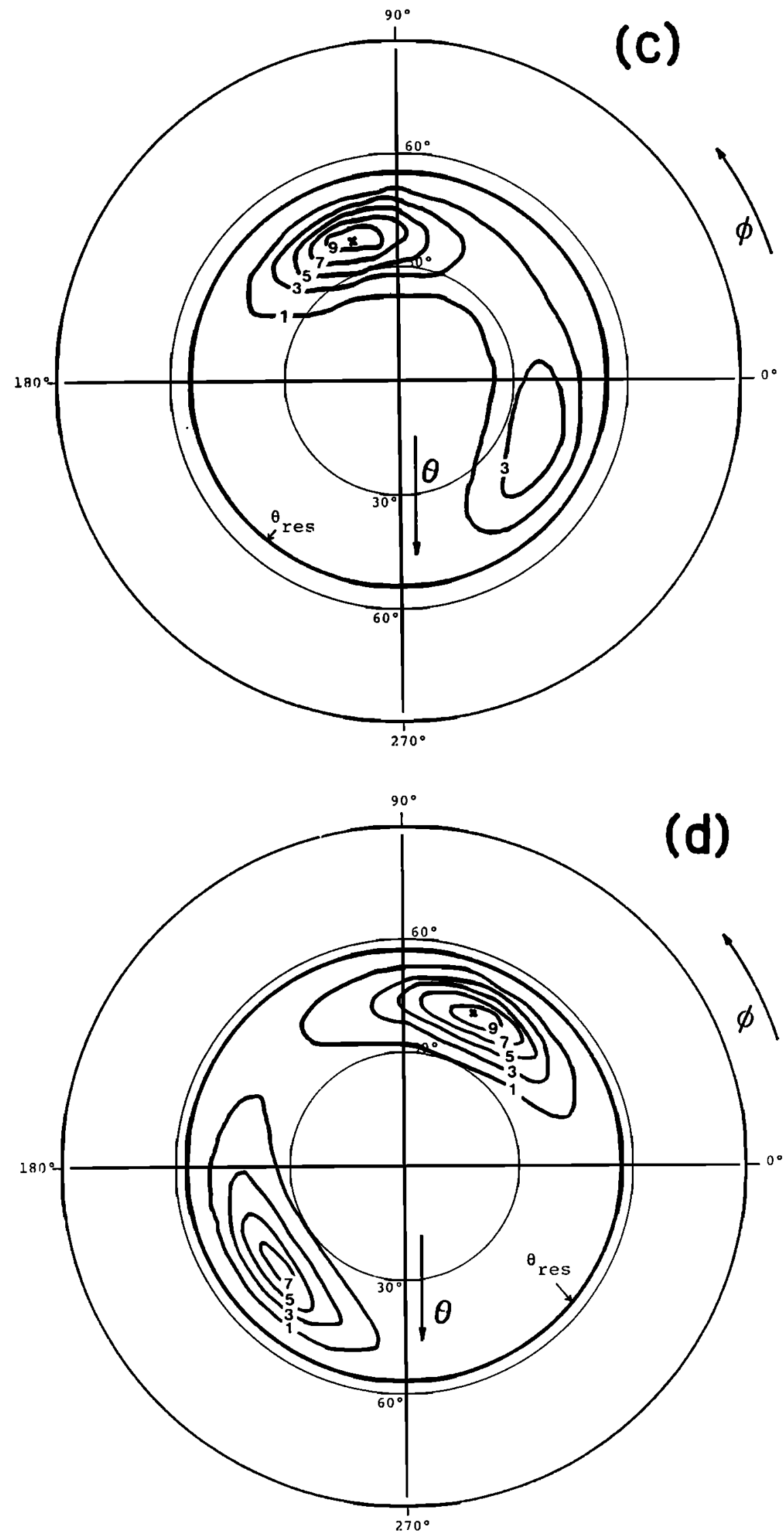

Fig. 4. (continued) 
(a)

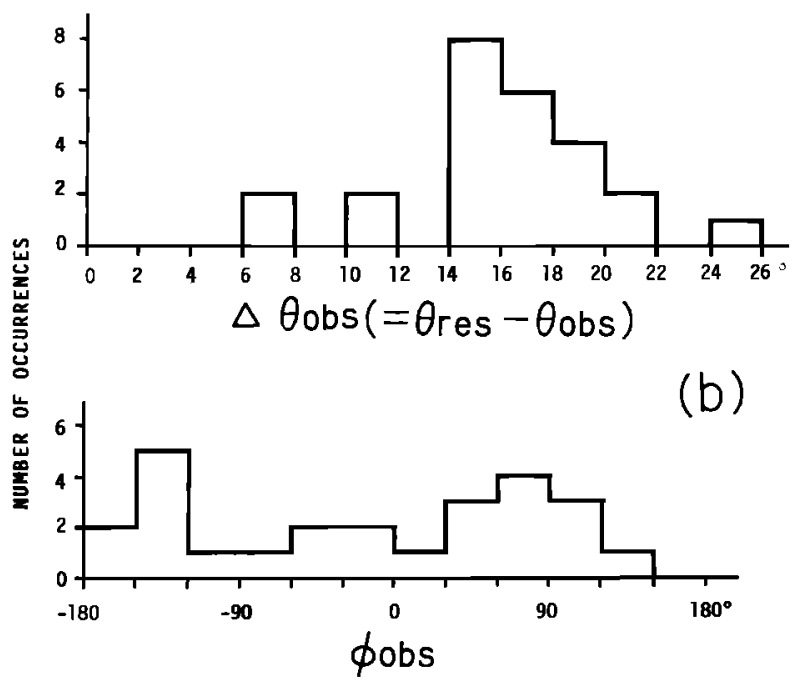

Fig. 5. The distribution of $\theta$ and $\phi$ values at the observing point, $\Lambda_{m} \sim 26^{\circ}:$ (a) the occurrence histogram of the difference between $\theta$ and $\theta_{\text {res }}$ and $(b)$ the occurrence histogram of the observed $\phi$ value.

4. The analyses show that the WDFs are double-peaked and that the wave energy tends to be mainly conveyed by waves whose $\theta$ values are very close to $\theta_{\text {res }}$ at the smaller latitude $\left(\Lambda_{m} \sim 17^{\circ}\right)$, whereas the $\theta$ values are farther from $\theta_{\text {res }}$ (about $15^{\circ}-20^{\circ}$ inside $\left.\theta_{\text {res }}\right)$ at the higher latitude $\left(\Lambda_{m} \sim 26^{\circ}\right)$. There seems to be no definite tendency in the $\phi$ distribution, the $\phi$ values being distributed over a large range. Finally, both the main and secondary peaks indicate that the waves have propagated to the observing points from the direction of the equator.

\section{Ray Tracing StUdies}

To estimate the wave normal directions of the upper band emissions at the geomagnetic equator, which is considered as a possible source region based on the experimental work of Hayakawa et al. [1984], we have carried out inverse ray tracing toward the equator, reversing the wave normal direction determined at the observation points as an input parameter. Before describing the results of the inverse ray tracing, it is interesting to report first on some direct ray tracing starting the waves at the equator, with their wave normals close to $\theta_{\text {res }}$, as found experimentally from Hayakawa et al. [1984] and theoretically suggested by Hashimoto and Kimura [1981] and Ohmi and Hayakawa [1986]. These direct ray tracing results will be shown to yield useful information on the propagation.

\subsection{Ray Tracing Method and Magnetospheric Model}

The ray tracing program used is a three-dimensional one developed by Cerisier [1970]. The ray paths are computed by a modified version of Haselgrove's [1954] ray tracing equations which is more suitable than the usual one for waves propagating near a resonance, as will be discussed later. The refractive index is calculated with the approximation that the (a)

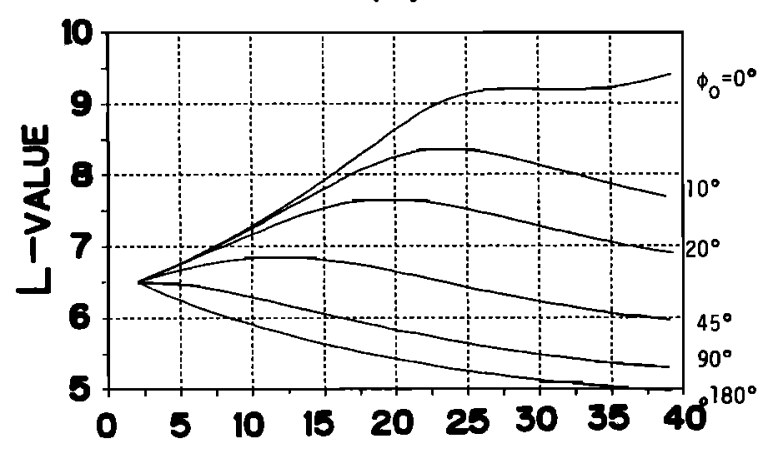

(c)

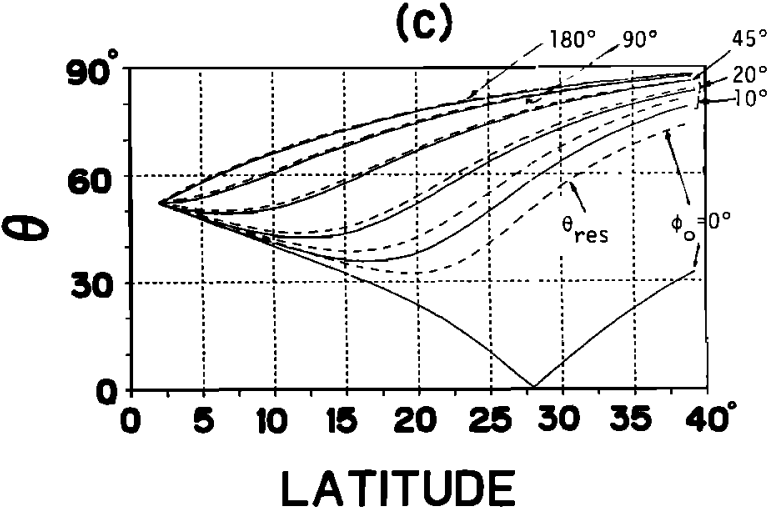

(b)
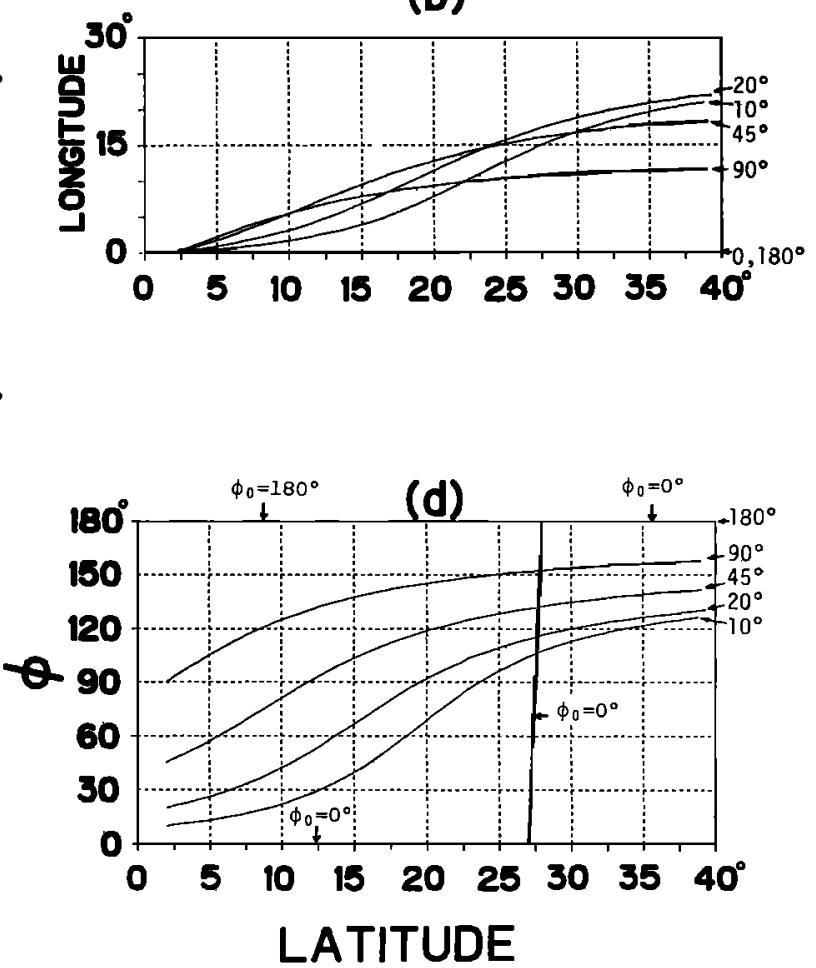

Fig. 6. The results of direct ray tracing with the initial conditions of $L_{0}=6.5, \Lambda=0.6, \mathrm{LAT}_{\mathrm{o}}=2.0^{\circ}$, and $\theta_{0}=\theta_{\text {res }}$ $-1.0^{\circ}$. The variation of ray paths: (a) $L$ versus LAT and (b) LONG versus LAT. The variation of wave normal direction along the ray path: (c) $\theta$ versus LAT ( $\theta_{\text {res }}$ is also indicated by the dashed lines) and (d) $\phi$ versus LAT. (Note: for $\phi_{0}=180^{\circ}$, $\phi$ remains $180^{\circ}$ along the path, but we have a discontinuous jump from $\phi=0^{\circ}$ to $\phi=180^{\circ}$ for $\phi_{0}=0^{\circ}$ in association with the corresponding discontinuous variation of $\theta$.) The parameter $\phi_{0}$ is the initial azimuthal direction. 

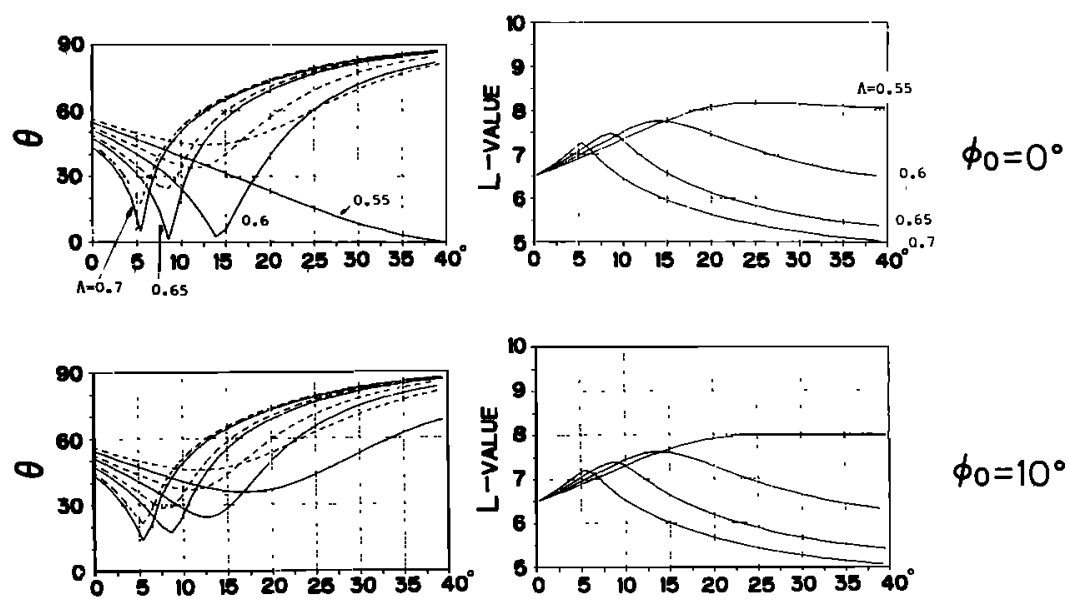

$$
\phi_{0}=10^{\circ}
$$
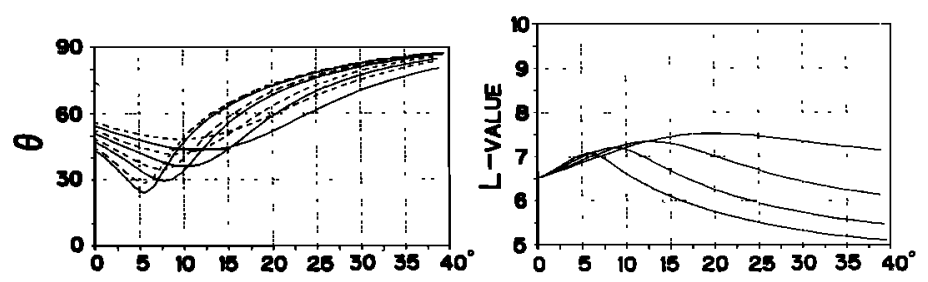

$\phi_{0}=20^{\circ}$
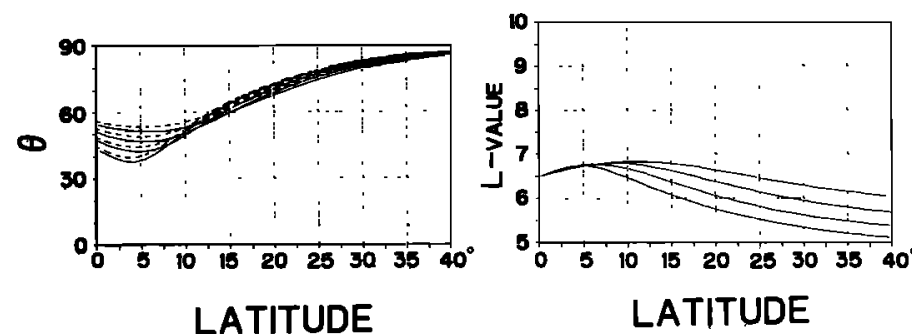

$\phi_{0}=45^{\circ}$

LATITUDE

Fig. 7. The effect of changing the wave frequency $\Lambda$ on the ray path and on the corresponding wave normal direction. Only the variations of $L$ (on the right) and of $\theta$ (on the left; $\theta_{\text {res }}$ as dashed lines again) with LAT are illustrated. $\operatorname{LAT}_{0}=0.0^{\circ}$ (equator), and the initial wave normal angle $\theta_{0}$ is changed here to $\theta_{\text {res }}-2.0^{\circ}$, to be compared with the results in Figure 6. The value of $\Lambda$ is only illustrated in the top panel for $\phi_{0}=0^{\circ}$, but one can easily find which curve refers to which $\Lambda$ because the general properties of the curves are the same for all $\phi_{0}$.

medium consists of a cold collisionless plasma composed of electrons and several species of ions. The density model is the same one defined by Cerisier [1970], which is derived from the isothermal diffusive equilibrium of electrons and positive ions [Angerami and Thomas, 1964]. Since the range of $L$ in which we are interested is $L=5.5-10$, the density model is adjusted so as to match the density as actually observed by satellites [Chappell, 1972]. The density is assumed to be $3.2 \mathrm{~cm}^{-3}$ at the geocentric distance $r$ of $43,317 \mathrm{~km}\left(r=6.8 R_{E} ; R_{E}\right.$ is the earth's radius), and the density variation in the equatorial plane in the range $6.5<L<7.75$ is about $r^{-3.6}$, in good agreement with the satellite measurement of Chappell [1972]. Longitudinal density gradients and duct structures are not considered. Finally, the geomagnetic field is assumed to be that of a centered dipole model.

In the following ray path results, the spatial location of the ray' is described by its geomagnetic latitude LAT, its geomagnetic longitude LONG, and its $L$ value. The propagation vector $\mathbf{k}$ is calculated at each point of the ray path, in a local Cartesian coordinate system $\mathrm{Oxyz}$, as defined in section 2 . The $k$ vector whose $\phi=0$ is oriented toward lower $L$ shells, whereas the one with $\phi=\pi$ is oriented toward higher $L$ shells. We denote by $\theta_{0}$ and $\phi_{0}$ the $\theta$ and $\phi$, values at the starting point of ray tracing.

\subsection{Direct Ray Tracing}

Figures $6 a$ and $6 b$ illustrates the variations of ray paths for waves started at $\mathrm{LAT}_{0}=2^{\circ}, L_{0}=6.5$, with an initial $\theta$ angle $\theta_{0}=\theta_{\text {res }}-1.0^{\circ}$ and a specific frequency, $\Lambda=0.6$. The variable parameter is the initial azimuthal angle $\phi_{0}$, which is varied from $0^{\circ}$ to $180^{\circ}$. The behavior for $\phi_{0}=180^{\circ}-360^{\circ}$ can be understood easily from the corresponding $\phi_{0}=Q^{\circ}-180^{\circ}$. The frequency is chosen to correspond to the main energy peak in the frequency spectrum observed by Hayakawa et al. [1984]. The initial latitude of $2.0^{\circ}$ is tentatively chosen because we have confirmed that the shift in initial latitude by about $5^{\circ}$ has no significant influence on the general tendencies, as will be presented in the following discussion.

The ray with $\phi_{0}=0^{\circ}$ is found as in Figure $6 a$ to propagate toward larger $L$ shells, because the initial $\mathbf{k}$ near the initial emission region is directed toward lower $L$ shells $\left(\phi_{0}=0^{\circ}\right)$, and the corresponding ray direction is directed in the $\phi=\pi$ 
(a)

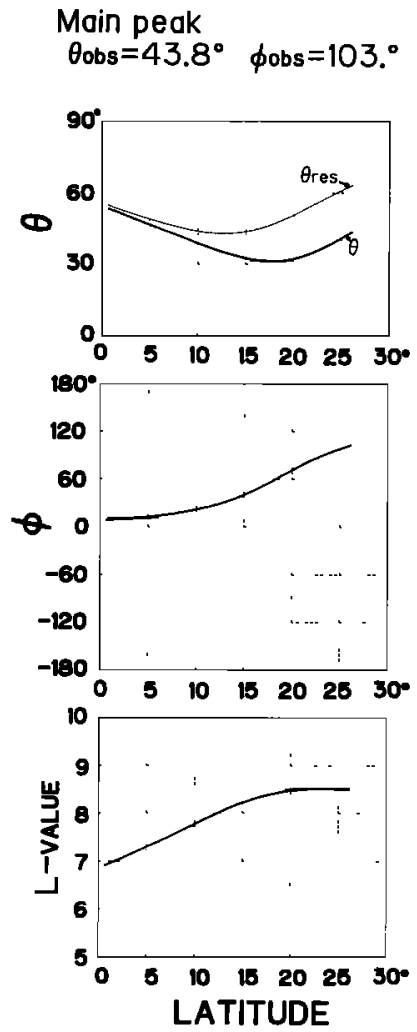

Secondary peak

$\theta_{0 b s}=46.2^{\circ}$ фobs $=-127$.
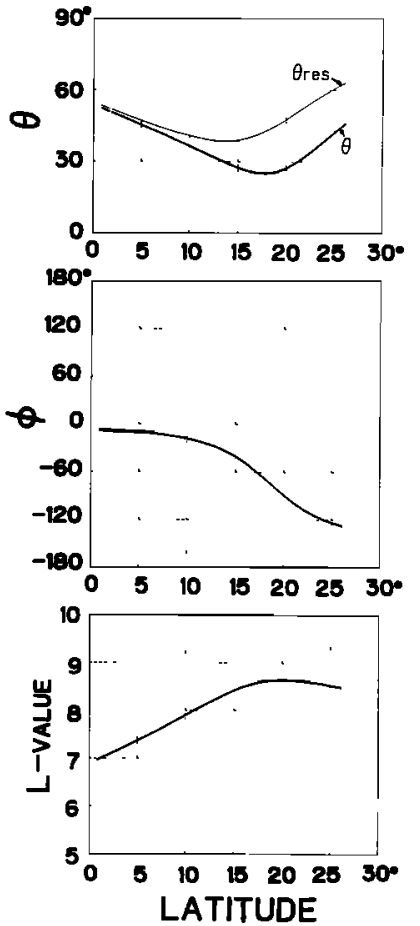

(b)

Main peak

$\theta$ obs $=46.2^{\circ}$ oobs $=126 .^{\circ}$
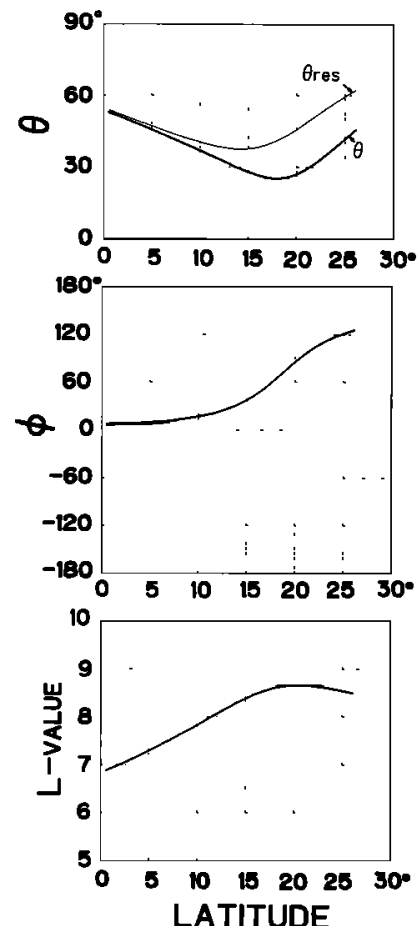

Secondary peok $\theta$ obs $=46.2^{\circ}$ фobs $=-4 .^{\circ}$
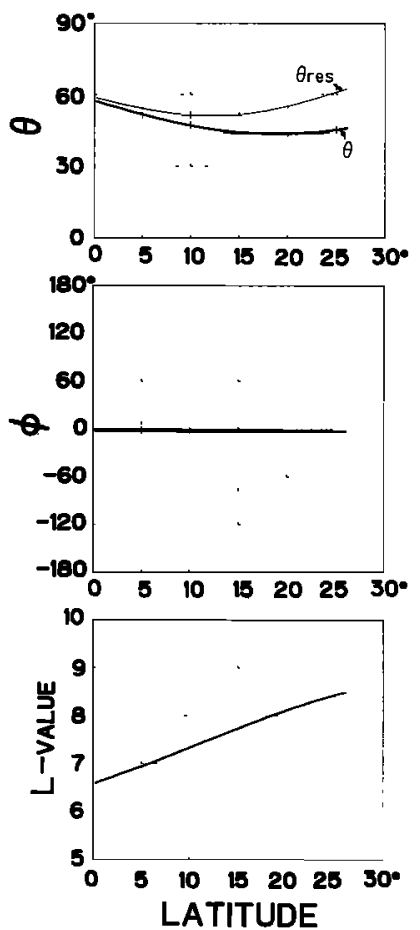

Fig. 8. The result of inverse ray tracing: (a) the result corresponding to the event of Figure $4 a$ and (b) the result corresponding to the event of Figure $4 b$. In each figure the left-hand panels refer to the main peak, while the right-hand panels refer to the secondary peak. The top and middle panels indicate the variations of wave normals $\theta$ and $\phi$ from the observing point to the equator, and the bottom panels the variations of the $L$ value of the ray path.

direction, as can be easily understood from the form of refractive index surface at the frequency $\Lambda=0.6$. As seen from Figure $6 a$, the ray with $\phi_{0}=0^{\circ}$ increases its $L$ value monotonically with LAT up to $\sim 25^{\circ}$, followed by a nearly constant $L$ value (9.2-9.5). For the ray with $\phi_{0}=20^{\circ}$ the $L$ value increases up to LAT $\sim 20^{\circ}$ and then decreases slowly with increasing LAT. The variation of $L$ value with LAT becomes less conspicuous for larger $\phi_{0}$ values, such as $45^{\circ}$. The rays with $\phi_{0}$ larger than $90^{\circ}$ exhibit a quite different behavior from those with $\phi_{0}<90^{\circ}$. The $L$ values of the former rays are seen to show a monotonic decrease. The variation of LONG with LAT is illustrated in Figure $6 b$; it shows that up to LAT $\sim 40^{\circ}$ the maximum deviation in LONG for a complete range of $0<\phi_{0}<\pi$ is about $20^{\circ}$.

Figures $6 c$ and $6 d$ illustrate the variations of wave normal directions $(\theta$ and $\phi)$ along the ray paths. The solid line refers to the $\theta$ variation with LAT, while the dashed line indicates the corresponding variation with LAT, of $\theta_{\text {res }}$ along the ray path for comparison with $\theta$. The features of the $\theta$ variation are very different in the two $\phi_{0}$ regions; that is, the $\theta$ variation when $\phi_{0}$ is close to $0^{\circ}$ is completely different from that for $\phi_{0}>10^{\circ}$. For $\phi_{0}=0^{\circ}$ the $\theta$ value decreases to depart from the $\theta_{\text {res }}$ slowly in the range of LAT $\leq 20^{\circ}$. Above that value there is a very significant departure from the local $\theta_{\text {res. }}$. For $\phi_{0}=10^{\circ}$ the difference $\left(\theta_{\text {res }}-\theta\right)$ slowly increases with LAT until a critical LAT $\left(\sim 17^{\circ}\right.$ in this case) and then becomes nearly constant. This tendency is confirmed for other larger values of $\phi_{0}$, with the following slightly different features: (1) the critical LAT becomes smaller with increasing $\phi_{0}$ up to $\phi_{0}=45^{\circ}$; for $\phi_{0} \gtrsim 45^{\circ}$, no critical latitude appears; and (2) $\left(\theta_{\text {res }}-\theta\right)$ becomes smaller with increasing $\phi_{0}$. The $\phi$ variation along the ray path is displayed in Figure $6 d$. The $\phi$ value tends to be collimated into the magnetic medium planes $\phi \sim 120^{\circ}-180^{\circ}$ during the course of propagation for all $\phi_{0} z$ $10^{\circ}$. Of course, as is easily understood from the figure, the wave with $\phi_{0}$ closer to $\phi_{0}=\pi$ tends to be collimated into the magnetic meridian plane earliest, and the wave with $\phi_{0} \sim 0^{\circ}$ takes a longer time (or larger LAT) to approach that plane. These characteristics in $\phi$ variation are the same as already found for smaller frequencies $(\Lambda<0.5)$ by Cairo and Lefeuvre [1986].

Although the results are not presented here, the general forms of ray paths are nearly the same as in Figures $6 a$ and $6 b$, when taking $L_{0}=6.08 .0$ in steps of $\Delta L_{0}=0.5$ for a fixed $\mathrm{LAT}_{0}$ in the range $0^{\circ}-5^{\circ}$, except that the curves are shilted. The dependence on $\mathrm{LAT}_{0}$ is such that the general characteristics are essentially unchanged, except that it takes more time for waves to be focused into the magnetic meridian plane for larger values of $\mathrm{LAT}_{0}$ such as $4^{\circ}-5^{\circ}$.

The most important parameters to have a significant influence on ray paths are the wave frequency $\Lambda$ and $\theta_{0}$. In order to examine this influence in greater detail, calculations have been performed with the following conditions: $L_{0}=6.5$; $\operatorname{LAT}_{0}=0^{\circ}$ (equator); $\theta_{0}=\theta_{\text {res }}-2.0^{\circ}$; and $\Lambda$ is varied from 0.55 to 0.7 in steps of 0.05 , for different $\phi_{0}$. The computational results are presented in Figure 7. The following features are pointed out.

1. In comparison with Figure $6 a$, where $\theta_{0}=\theta_{\text {res }}-1.0^{\circ}$, 

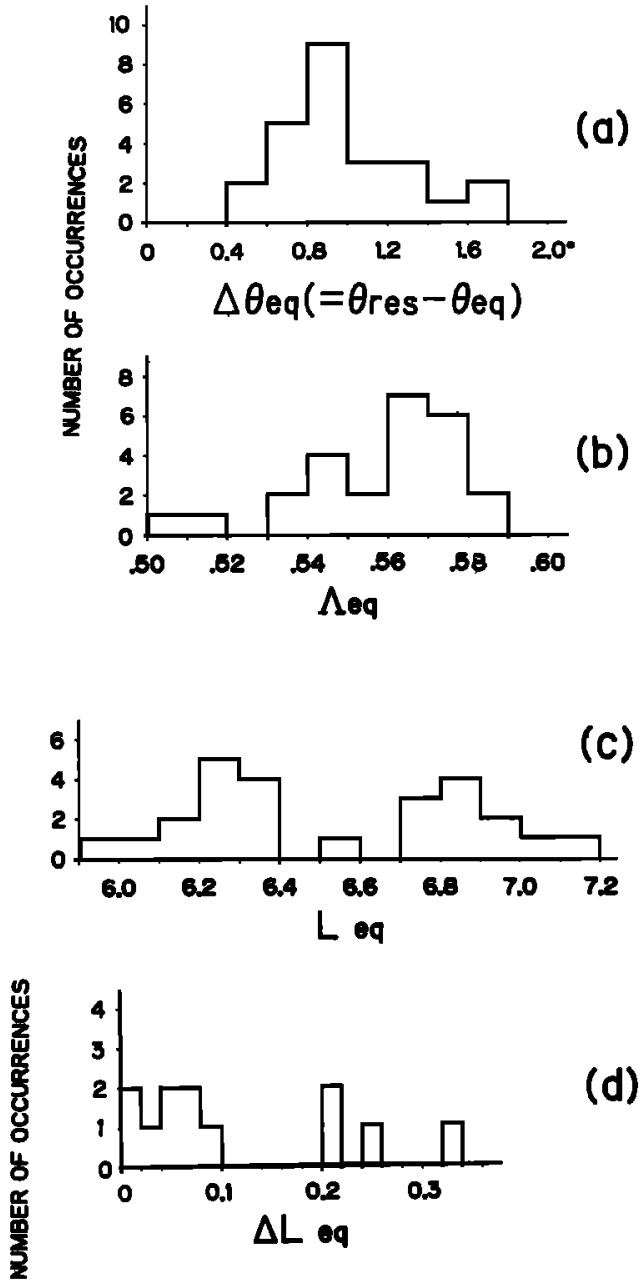

(d)

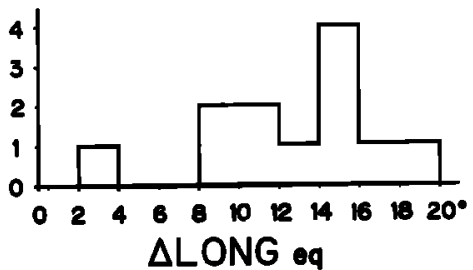

(e)

Fig. 9. Summary of the results of inverse ray tracing studies for the event of September 19, 1977. (a) The occurrence histogram of $\theta$ at the equator, in the form of $\Delta \theta=\theta_{\text {res(eq) }}-\theta_{\text {eq. }}$ (b) The occurrence histogram of the normalized wave frequency $\Lambda_{e q}$ at the equator. (c) The occurrence histogram of $L_{\text {eq }}$. These figures are based on all the main and secondary peaks. $(d)$ The occurrence histogram of the difference in $L_{e q}$ for the main and secondary peaks for each case. $(e)$ The corresponding difference in LONG $\left(\Delta \mathrm{LONG}_{\mathrm{eq}}\right)$.

the departure in $L$ from $L_{0}$ becomes smaller for this smaller $\theta_{0}$ $\left(=\theta_{\text {res }}-2.0^{\circ}\right)$ for the same $\Lambda$ and $\phi_{0}$. Namely, the wave with wave normal closer to $\theta_{\text {res }}$ tends to suffer a greater traversal, with a larger departure in $L$ from $L_{0}$, as would be expected.

2. The general characteristics of the $\theta$ variation are the same whatever $\Lambda$ is, but the increase in $\Lambda$ shifts the curves toward smaller LAT.

\subsection{Inverse Ray Tracing}

In this section we perform inverse ray tracing toward the equator, which is considered to be the source region, using the inverted observed wave normals at the observation positions as the starting wave normals. Inverse ray tracing has been carried out for the events on October 12 and September 19, 1977 (Table 1).

Figures $8 a$ and $8 b$ represent two examples of the results of inverse ray tracings for the events shown in Figures $4 a$ and $4 b$, respectively. In each figure the left-hand three panels refer to the main peak, and the right to the corresponding secondary peak. The top and middle panels represent the variations of wave normal direction $\mathbf{k}(\theta$ and $\phi$ ) during the propagation from the observation point to the equator, and the bottom panels indicate the variation of $L$ value. In the top panels the local $\theta_{\text {res }}$ along the ray path is also plotted for comparison. Let us examine, in detail, Figure $8 a$. For both main and secondary peaks, $\theta$ is found to approach the local $\theta_{\text {res }}$ toward the equator, and the $\theta$ value at the equator is only a few degrees inside $\theta_{\text {res }}$. The corresponding $\phi$ variation indicates that the $\phi$ values approach $\phi \sim 0$ for both peaks. Hence the result of inverse ray tracing is in accord with the corresponding direct ray tracing result with $\phi_{0} \sim 10^{\circ}$ (see Figure $6 a$ ). The $L$ value at the equator is found to be about 6.8 for both main and secondary peaks. Although not shown, the difference in LONG at the equator for the two peaks is about $17^{\circ}$. Note that the equatorial normalized frequency is $\Lambda_{e q}=0.567$ for the main peak and $\Lambda_{\text {eq }}=0.578$ for the secondary peak, while the normalized frequency at the observing point is $\Lambda=0.432$ for both peaks. Very similar variations in $\theta, \phi$, and $L$ are recognizable for the main and secondary peaks in Figure $8 b$. The equatorial normalized frequency is 0.577 for the main peak and 0.516 for the secondary peak, while $\Lambda=0.446$ at the observing point.

The summary of inverse ray tracing results for all events on both days is given in Figure 9. Figure $9 a$ gives the occurrence histogram of the $\theta$ value with respect to $\theta_{\text {res }}$ at the equator, Figure $9 b$ that of the normalized frequency $\Lambda_{\text {eq }}$, Figure $9 c$ that of the $L$ value at the equator, Figure $9 d$ the difference in $L_{\text {eq }}$ for the main and secondary peaks for each event, and Figure $9 e$ the occurrence histogram of the difference in LONG for the two peaks for each event. Figure $9 a$ implies definitely that the value of the upper band emissions is always very close to $\theta_{\text {res }}$ at the equator. Figure $9 b$ indicates that the wave frequency seems to be always above one-half the equatorial electron gyrofrequency (the wave frequency is randomly selected from the whole range in the upper band spectra, and so the minimum frequency in Figure $9 b$ should be of significance). Figures $9 d$ and $9 e$ show that the source region of the main and secondary peaks for each event is very limited in $L_{e q}$ (of the order of $\Delta L_{\text {eq }} \sim 0.3$ at maximum) but rather extended in LONG $\left(\Delta \mathrm{LONG}_{\text {eq }} \lesssim 20^{\circ}\right.$ ). Such source regions are expected to be located in the range of $L_{\mathrm{eq}}=6.0-7.2$ as in Figure $9 \mathrm{c}$.

\subsection{Summary of Ray Tracing Studies}

The following facts have emerged from the inverse ray tracing studies in combination with the direct ray tracing results:

1. The wave normal direction $\theta$ of the upper band VLF emissions (both main and secondary peaks), traced backward to the equator, is found to be in the vicinity of $\theta_{\text {res }}$. The normalized frequency $\Lambda_{\text {eq }}$ is always above 0.5 . The $\phi$ value in the equatorial region is around the meridian plane $\left( \pm 5^{\circ}\right)$. Since the number of observing points is very limited, the $\phi$ information is not as important, because the observed $\phi$ value is strongly dependent on the relative location between the observing point and the source region. The variation of $\mathbf{k}$ from the observing point toward the equator confirms the model of a wave generation at $\theta$ close to $\theta_{\text {res }}$. 
2. The inverse ray tracing results for the main and secondary peaks for all events have yielded that the difference in $L_{\mathrm{eq}}$ is relatively small, $\Delta L_{\mathrm{eq}} \lesssim 0.3$, but the difference in LONG $_{\mathrm{eq}}$ is rather large, $\lesssim 20^{\circ}$, suggesting a source region well elongated in longitude. Such source regions are located in two $L$ regions, $L_{\text {eq }}=6.0-6.4$ and $L_{\text {eq }}=6.7-7.2$ for the events studied here.

\section{Comparison of the Ray Tracing Results WITH THE OBSERVATIONS AND Discussion of Wave Generation and Propagation}

In this section we discuss the comparison between the observational facts (wave spectra and wave normal directions) in section 3 and the theoretical direct ray tracing and inverse ray tracing started with the wave normal directions determined in the off-equatorial regions.

The most important conclusions derived from the inverse ray tracings are summarized in section 4.4: (1) the $\theta$ values in the equatorial region are close to $\theta_{\text {res }}$ and (2) $\Lambda_{\text {eq }}$ is above 0.5 . Point 2 is strongly indicative of a generation of the upper band VLF emissions in the vicinity of the equator. This result is consistent with our previous direction finding, made exactly at the equator [Hayakawa et al., 1984], which indicated that upper band VLF emissions appear mainly above one-half the equatorial gyrofrequency $\left(\omega / \omega_{H} \gtrsim 0.5\right)$. Point 1 seems to lend further support to our model of wave generation in the quasielectrostatic model [Hayakawa et al., 1984]. As a result we can conclude that the upper band VLF emissions analyzed here can be identified with confidence as being "half gyrofrequency" VLF emissions, excited just around the equator, with their wave normals close to $\theta_{\text {res }}$. It is a propagation effect which places the frequency gap at frequencies smaller than half the gyrofrequency at the observing points. Furthermore, it should be noted that the lower band chorus emissions are also believed to be generated in those source regions by higherenergy hot electrons [Hayakawa et al., 1984].

As a generation mechanism of the "half-gyrofrequency" VLF emissions, Ohmi and Hayakawa [1986] have proposed a theory which is briefly described below. Recent satellite measurements of energetic particles [Bahnsen et al., 1985] have shown that the energetic electron (hot electron) data in the equatorial plane at $L \sim 6.6$ could be fitted, on many occasions, by a sum of two Maxwellian distributions with temperatures of $\sim 100 \mathrm{eV}$ and a few $\mathrm{keV}$. The densities as well as energy fluxes vary strongly with geomagnetic activity, while the cold plasma density is relatively stable. Hot electrons are expected to have a temperature anisotropy, and Ohmi and Hayakawa [1986] have proposed a plasma condition that consists of cold electrons and low-energy (suprathermal) hot electrons with a temperature of the order of a few tens of electron volts, which can be unstable to half-gyrofrequency waves in terms of an electrostatic electron cyclotron (Harristype) instability. The theoretical prediction by Ohmi and Hayakawa [1986] is found to be in good agreement with the observational facts in the present paper. It should also be added that when the population of higher-energy electrons with a temperature of a few $\mathrm{keV}$ is increased, we can expect the excitation of lower band chorus emissions as in Figures 1 and 3 in terms of an electromagnetic electron cyclotron instability [Hayakawa et al., 1984].

Finally, we comment on the bandwidth of the upper band emissions because it is closely related to the generation mechanism. As summarized in section 3.3, the bandwidth of the upper band in the present paper is nearly of the same magnitude as that of our previous equatorial observation [Hayakawa et al., 1984], while Coroniti et al. [1971] observed a very narrow band off the equator $\left(\Lambda_{m} \sim 10^{\circ}\right)$. Hashimoto and Kimura [1981] and Ohmi and Hayakawa [1986] have suggested that emissions which propagate to high latitudes should be of narrow bandwidth, like those of Coroniti et al. [1971]), if we assume a relatively wide generation region within which the temperature anisotropy decreases significantly with increasing latitude. However, as is found in section 5, the generation region estimated in this paper is considered to be localized to a narrow region around the equator where the change of temperature anisotropy is negligibly small, so that the bandwidth of the upper band emissions is mainly determined by the generation characteristics around the equator. So the discrepancy in the observed bandwidth may possibly be due to the different characteristics of the source region.

Acknowledgments. The authors would like to express their sincere thanks to J. Etcheto and B. Higel of CRPE (Issy-les-Moulineaux) and P. M. E. Decreau of LPCE (Orleans) for supplying the data of plasma density measurements from the S-301 and S-304 experiments, respectively. Thanks are also due to CNES (Toulouse) for making the spectrograms on microfiches, some of which have been reproduced in the present paper. We are grateful to J. C. Cerisier (CRPE, Saint Maur) for permitting us to use his three-dimensional ray tracing program and L. Cairo of LPCE (Orleans) for his useful comments on the ray tracing. Useful discussions with J. Ohtsu, Y. Tanaka, and T. Okada of Nagoya University, S. Shimakura of Chiba University, and C. Beghin of LPCE (Orleans) are greatly appreciated. The present work is partly supported by the International Joint Program of Japan Society of Promotion of Science and also partly by Takeda Science Foundation. One of the authors (M.H.) is thankful to A. Kimpara, emeritus professor of Nagoya University, for his continuous encouragement. Finally, we thank the two referees for their very useful criticisms.

The Editor thanks J. L. Roeder and another referee for their assistance in evaluating this paper.

\section{REFERENCES}

Angerami, J. J., and J. O. Thomas, Studies of planetary atmospheres, 1 , The distribution of electrons and ions in the earth's exosphere, $J$. Geophys. Res., 69, 4537, 1964.

Bahnsen, A., M. Jespersen, T. Neubert, P. Canu, H. Borg, and P. E. Frandsen, Morphology of keV-electrons in the Earth's magnetosphere as observed by GEOS 1, Ann. Geophys., Gauthier Villars, 3, 19, 1985.

Buchalet, L. J., and F. Lefeuvre, One and two-direction models for VLF electromagnetic waves observed on board GEOS 1, J. Geophys. Res., 86, 2377, 1981.

Burtis, W. J., and R. A. Helliwell, Magnetospheric chorus: Occurrence patterns and normalized frequency, Planet. Space Sci., 24, 1007, 1976.

Cairo, L., and F. Lefeuvre, Localization of sources of ELF/VLF hiss observed in the magnetosphere: Three-dimensional ray tracing, $J$. Geophys. Res., 91, 4352, 1986.

Cerisier, J. C., Propagation perpendiculaire au voisinage de la fréquence de la résonance hybride basse, in Plasma Waves in Space and in the Laboratory, vol. 2, edited by $\mathbf{J}$. O. Thomas and B. J. Landmark, p. 487, Edinburgh University Press, Edinburgh, 1970.

Chappell, C. R., Recent satellite measurements of the morphology and dynamics of the plasmasphere, Rev. Geophys., 10, 951, 1972.

Coroniti, F. V., R. W. Fredricks, C. F. Kennel, and F. L. Scarf, Fast time resolved spectral analysis of VLF banded emissions, J. Geophys. Res., 76, 2366, 1971.

Haselgrove, J., Ray theory and a new method for ray tracing, in The Physics of the Ionosphere, p. 355, Physical Society, London, 1954.

Hashimoto, K., and I. Kimura, A generation mechanism of narrowband hiss emissions above one-half the electron cyclotron frequency in the outer magnetosphere, $J$. Geophys. Res., 86, 11,148, 1981.

Hayakawa, M., Y. Yamanaka, M. Parrot, and F. Lefeuvre, The wave 
normals of magnetospheric chorus emissions observed on board GEOS 2, J. Geophys. Res., 89, $2811,1984$.

Hayakawa, M., N. Ohmi, M. Parrot, and F. Lefeuvre, Direction finding of ELF hiss emissions in a detached plasma region of the magnetosphere, J. Geophys. Res., 91, 135, 1986.

Helliwell, R. A., Whistlers and Related Ionospheric Phenomena, p. 30, Stanford University Press, Stanford, Calif., 1965.

Jones, D., Introduction to the S-300 wave experiments onboard GEOS, Space Sci. Rev., 22, 327, 1978.

Kennel, C. F., and M. Ashour-Abdalla, Electrostatic waves and the strong diffusion of magnetospheric electrons, in Magnetospheric Plasma Physics, edited by A. Nishida, p. 297, Centre for Academic Publications, Tokyo, 1982.

Kennel, C. F., F. L. Scarf, R. W. Fredricks, J. H. Geehee, and F. V. Coroniti, VLF electric field observations in the magnetosphere, $J$. Geophys. Res., 75, 6136, 1970.

Knott, K., The GEOS-1 mission, Space Sci. Rev., 22, 321, 1978.

Lefeuvre, F., and R. A. Helliwell, Characterization of the sources of VLF hiss and chorus observed on GEOS 1, J. Geophys. Res., 90, 6419, 1985.

Lefeuvre, F., M. Parrot, and C. Delannoy, Wave distribution function estimation of VLF electromagnetic waves observed on board GEOS 1, J. Geophys. Res., 86, 2359, 1981.

Lefeuvre, F., T. Neubert, and M. Parrot, Wave normal directions and wave distribution functions for ground-based transmitter signals observed on GEOS 1, J. Geophys. Res., 87, 6203, 1982.
Maeda, K., Cyclotron side-band emissions from ring current electrons, Planet. Space Sci., 24, 341, 1976.

Ohmi, N., and M. Hayakawa, On the generation of halfgyrofrequency VLF emissions in the magnetospheric plasma, $J$. Plasma Phys., 35, 351, 1986.

Parrot, M., and F. Lefeuvre, Statistical study of the propagation characteristics of ELF hiss observed on GEOS 1, inside and outside the plasmapause, Ann. Geophys., Gauthier Villars, 5, 363, 1986.

S-300 Experimenters, Measurements of electric and magnetic wave fields and of cold plasma parameters onboard GEOS-1, Preliminary results, Planet. Space Sci., 27, 317, 1979.

Tsurutani, B. T., and E. J. Smith, Postmidnight chorus: A substorm phenomenon, J. Geophys. Res., 79, 118, 1974.

M. Hayakawa and H. Muto, Research Institute of Atmospherics, Nagoya University, 13 Honohara 3-chome, Toyokawa, Aichi, 442 Japan.

F. Lefeuvre and M. Parrot, Laboratoire de Physique et Chimie de l'Environnement, Centre National de la Recherche Scientifique, 45071 Orléans Cedex 2, France.

(Received December 17, 1986;

revised March 26, 1987;

accepted March 12, 1987.) 